\title{
Radiomic features of primary tumor by lung cancer stage: analysis in BRAF mutated non-small cell lung cancer
}

\author{
Atul Padole ${ }^{1,2 \#}$, Ramandeep Singh ${ }^{1,2 \#}$, Eric W. Zhang ${ }^{1,2}$, Dexter P. Mendoza ${ }^{1,2}$, Ibiayi Dagogo-Jack ${ }^{2,3}$, \\ Mannudeep K. Kalra ${ }^{1,2}$, Subba R. Digumarthy ${ }^{1,2}$ \\ ${ }^{1}$ Division of Thoracic Imaging, Massachusetts General Hospital, Boston, MA, USA; ${ }^{2}$ Harvard Medical School, Boston, MA, USA; ${ }^{3}$ Cancer Center, \\ Department of Medicine, Massachusetts General Hospital, Boston, MA, USA \\ Contributions: (I) Conception and design: SR Digumarthy, A Padole, R Singh; (II) Administrative support: A Padole, R Singh, EW Zhang, DP \\ Mendoza; (III) Provision of study materials or patients: SR Digumarthy, I Dagogo-Jack; (IV) Collection and assembly of data: All authors; (V) Data \\ analysis and interpretation: All authors; (VI) Manuscript writing: All authors; (VII) Final approval of manuscript: All authors. \\ \#These authors contributed equally to this work. \\ Correspondence to: Dr. Subba R. Digumarthy, MD. Division of Thoracic Imaging and Intervention, Massachusetts General Hospital, 55 Fruit Street, \\ Founders 202, Boston, MA 02114, USA. Email: sdigumarthy@mgh.harvard.edu.
}

\begin{abstract}
Background: The clinical features and traditional semantic imaging characteristics of $B R A F$-mutated nonsmall cell lung cancer (NSCLC) have been previously reported. The radiomic features of $B R A F$-mutated NSCLC and their role in predicting cancer stage, however, have yet to be investigated. This study's goal is to assess the differences in CT radiomic features of primary NSCLC driven by BRAF mutation and stratified by tumor-node-metastasis (TNM) staging.

Methods: Our IRB approved study included 62 patients with BRAF mutations (V600 in 27 and non-V600 in 35 patients), who underwent contrast-enhanced chest CT. Tumor stage was determined based on the $8^{\text {th }}$ edition of TNM staging. Two thoracic radiologists assessed the primary tumor imaging features such, including tumor size (maximum and minimum dimensions) and density (Hounsfield units, HU). Deidentified transverse CT images (DICOM) were processed with 3D slicer (Version 4.7) for manual lesion segmentation and estimation of radiomic features. Descriptive statistics, multivariate logistic regression, and receiver operating characteristics (ROC) were performed.

Results: There were significant differences in the radiomic features based on cancer stages I-IV with the most significant differences between stage IV and stage I lesions [AUC 0.94 (95\% CI: 0.86-0.99), P<0.04]. There were also significant differences in radiomic features between stage IV and combined stages I-III [40/113 radiomic features; AUC 0.71 (95\% CI: 0.59-0.85); $\mathrm{P}<0.04-0.0001$ ]. None of the clinical (0/6) or imaging (0/3) features were significantly different between stage IV and combined stages I-III.

Conclusions: The radiomic features of primary tumor in BRAF driven NSCLC significantly vary with cancer stage, independent of standard imaging and clinical features.
\end{abstract}

Keywords: BRAF; lung cancer; multidetector row computed tomography; radiomics

Submitted Feb 28, 2020. Accepted for publication Jul 13, 2020.

doi: $10.21037 /$ tlcr-20-347

View this article at: http://dx.doi.org/10.21037/tlcr-20-347

\section{Introduction}

Lung malignancy is the most frequent cause of cancerrelated mortality in the world, accounting for approximately $24 \%$ of all cancer-related deaths in the United States (1).
Tumor size, a readily assessed feature on medical imaging, is a key determinant in the staging of lung cancer, with many studies correlating increasing tumor size with decreasing overall survival $(2,3)$. Unsurprisingly, most changes in the recently revised tumor-node-metastasis (TNM) 
classification for lung cancer are based on the largest tumor dimension $(4,5)$.

Converging evidence from the biologic and genetic sciences suggests that size alone is insufficient to explain the progression of lung cancer. Small primary tumors $(<5 \mathrm{~mm})$ can demonstrate a remarkable ability to metastasize to local lymph nodes and distant organs (6), thus supporting the hypothesis that lung cancers are not uniform in their biologic or genetic make-up. Indeed, genetic analyses of lung cancers have revealed different genetic and morphologic phenotypes both within a tumor and across different tumors $(7,8)$. Co-existence of lung cancers with non-invasive tumors cells, metastatic tumor cells, or a polyclonal population of both offers a compelling explanation for the co-existence of an early $\mathrm{T}$ stage and an overall stage IV tumor (9).

CT helps guide clinical decision-making related to diagnosis, staging, and treatment-response in lung cancer $(10,11)$. Traditional semantic imaging characteristics help establish the TNM stage but cannot differentiate between different clonal populations of lung cancer. Several studies suggest that this limitation can be addressed with radiomic features (12). Extraction of sub-visual and quantifiable data from imaging modalities such as CT and MRI with radiomic features can help create cancer predictive and prognostic correlations. Recent studies report that radiomic features can distinguish between well- and poorly-differentiated lung cancers, categorize benign and malignant subsolid nodules, assess tumor prognosis and treatment response, and predict underlying lung cancer histology (12-17). However, the utility of radiomic features is unclear in differentiating or predicting lung cancer stage based on differences in underlying tumor clonal populations.

Patients with BRAF-mutated non-small cell lung cancer (NSCLC) were included in the study in order to have a homogeneous study population and minimize any confounders related to different driver mutations of lung cancer and different histologic subtypes. BRAF mutations are a rare, but potentially targetable mutation in the treatment of NSCLC (18). BRAF is a protein kinase in the RAS/MAPK pathway, which can lead to cell proliferation and oncogenesis when constitutively activated (19). The clinical features and traditional semantic imaging characteristics of $B R A F$-mutated NSCLC have been previously reported $(19,20)$. To our knowledge, however, the radiomic features of $B R A F$-mutated NSCLC and the role of these radiomic features in predicting cancer stage have yet to be investigated. To address these knowledge gaps, we assessed the role of radiomic features to differentiate between different TNM stages (I-IV) of $B R A F$-mutated NSCLC.

We present the following article in accordance with the MDAR and STROBE reporting Checklist (available at http://dx.doi.org/10.21037/tlcr-20-347).

\section{Methods}

The study was conducted in accordance with the Declaration of Helsinki (as revised in 2013). This study was reviewed and approved by the Partners Human Research Committee (Institutional review board protocol numbers 2019P000198, 2016P000767). Informed consent was waived by the IRB due to retrospective nature of the study.

\section{Patient characteristics}

Our hospital thoracic oncology database between 20052017 was searched to identify $B R A F$-mutant NSCLC. The presence of $B R A F$ mutation was confirmed with either $\mathrm{SNaPshot}$, a technique that uses next generation sequencing to interrogate $B R A F$ exons 11 and 15 , or polymerase chain reaction technique for those diagnosed before 2014. This search yielded 105 patients who had pre-treatment scans available for review. After excluding 43 patients who had only attenuation correction CT performed as part of CTPET imaging and those with CT imaging without IV contrast, 62 patients with diagnostic contrast enhanced CT scans were selected for the study. The included scans were without motion or beam hardening artifacts that could limit the assessment of lung cancer. The specific BRAF mutations were V600 in 27 and non-V600 in 35 patients. The functional classes (21) of $B R A F$ mutant NSCLC were: class 1 in 27/62, class 2 in 22/62 and class 3 in 13/62 patients (Figure 1). The demographics and clinical features such as gender, age, race, histology, smoking history and duration of smoking were extracted from patient electronic medical records.

\section{CT scan parameters}

The CT examinations were performed on multidetectorrow CT scanners (MDCT) from various vendors using automatic exposure control or fixed $\mathrm{mA}$ at a tube potential of $100-120 \mathrm{kV}$. Images were acquired after administration of intravenous iodinated contrast with standard oncology 


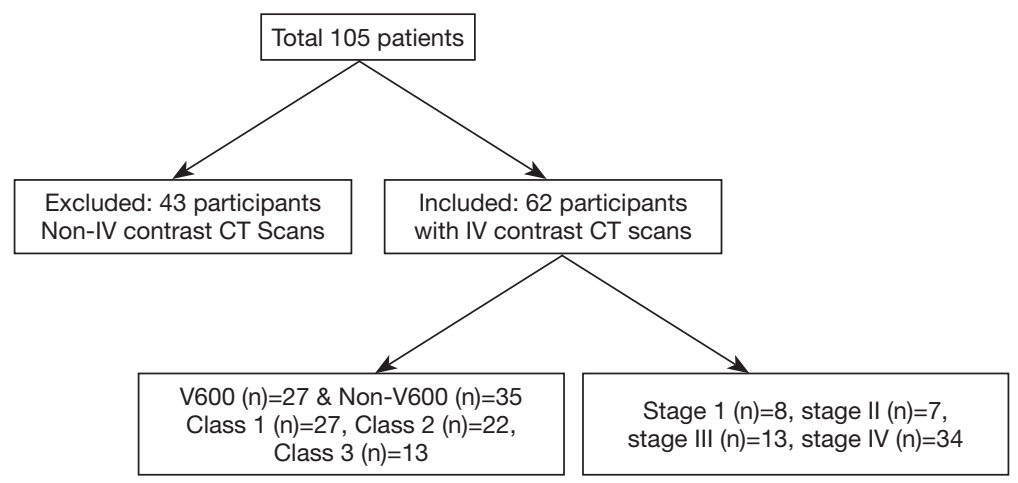

Figure 1 Flow-chart diagram showing the inclusion of patients in the study.

imaging protocol. Image reconstruction was performed at slice thickness of 1-3 $\mathrm{mm}$ for chest using standard soft tissue reconstruction kernels and at a minimum of $5 \mathrm{~mm}$ for abdomen and pelvis with images also reconstructed at sagittal and coronal planes. When available brain MRI, head CT, and FDG-PET images were reviewed to determine the stage of lung cancer.

\section{Image analysis}

The imaging studies were reviewed by two thoracic radiologists (DPM and SRD) with 1 and 17 years of experience. The images were assessed concurrently and in consensus. The primary tumor assessment included size, location, density (solid, mixed, ground-glass), cavitation, air bronchogram and calcification. The stage of the tumor was determined based on the primary tumor, nodal disease and other sites of disease with in the thorax and outside the thorax based on TNM $8^{\text {th }}$ edition. The histological confirmation was considered gold standard for nodes and metastases when available. Otherwise FDG avidity on PET scan and standard CT imaging features such as nodes greater than $1 \mathrm{~cm}$ in short axis and interval growth were taken as proof of disease. The measurements on standard imaging features included maximum and minimum tumor dimensions and lesion density (mean Hounsfield unit, $\mathrm{HU})$.

\section{Radiomic features}

The transverse CT images [de-identified transverse CT images (DICOM)] were de-identified and exported offline from the Picture Archiving and Communication System archives and were processed using 3D slicer (Version 4.7), an open source software package. Manual segmentation of area for each lesion was performed using the paint function on chest CT images in lung window (Figure 2). The images were contoured with $3 \mathrm{D}$ slicer and we applied the resampling to isotropic voxel size and we used the default discretization of voxel bins. The original radiomic features $(n=113)$ were extracted using radiomics applet. The first order features include mean, median, standard deviation, maximum, minimum, entropy (randomness), and skewness and kurtosis. The second-order features include GrayLevel Co-Occurrence Matrix (GLCM), Gray-Level RunLength Matrix (GLRLM), Gray Tone Difference Matrix (GTDM) and Grey Level Size Zone Matrix (GLSZM). Data were exported to Microsoft EXCEL (Microsoft Inc., Redmond, Washington). A study co-investigator (AP) made all measurements in consultation with a fellowshiptrained thoracic radiologist (SRD, 17 years of experience). The detailed description of the radiomic features is available on https://pyradiomics.readthedocs.io/en/latest/ features.html.

\section{Statistical analysis}

Data were analyzed using SPSS 21 statistical software (IBM, Armonk, NY). Pearson correlation coefficient was calculated for clinical, imaging and radiomic features. Univariate and multivariate logistic regression was performed to determine the most significant features. To avoid the multiple collinearity, the variance inflation factor (VIF) was calculated with linear regression analysis. Only, predictors with VIF of less than 5 were selected to determine the significance of radiomic features. Receiver 

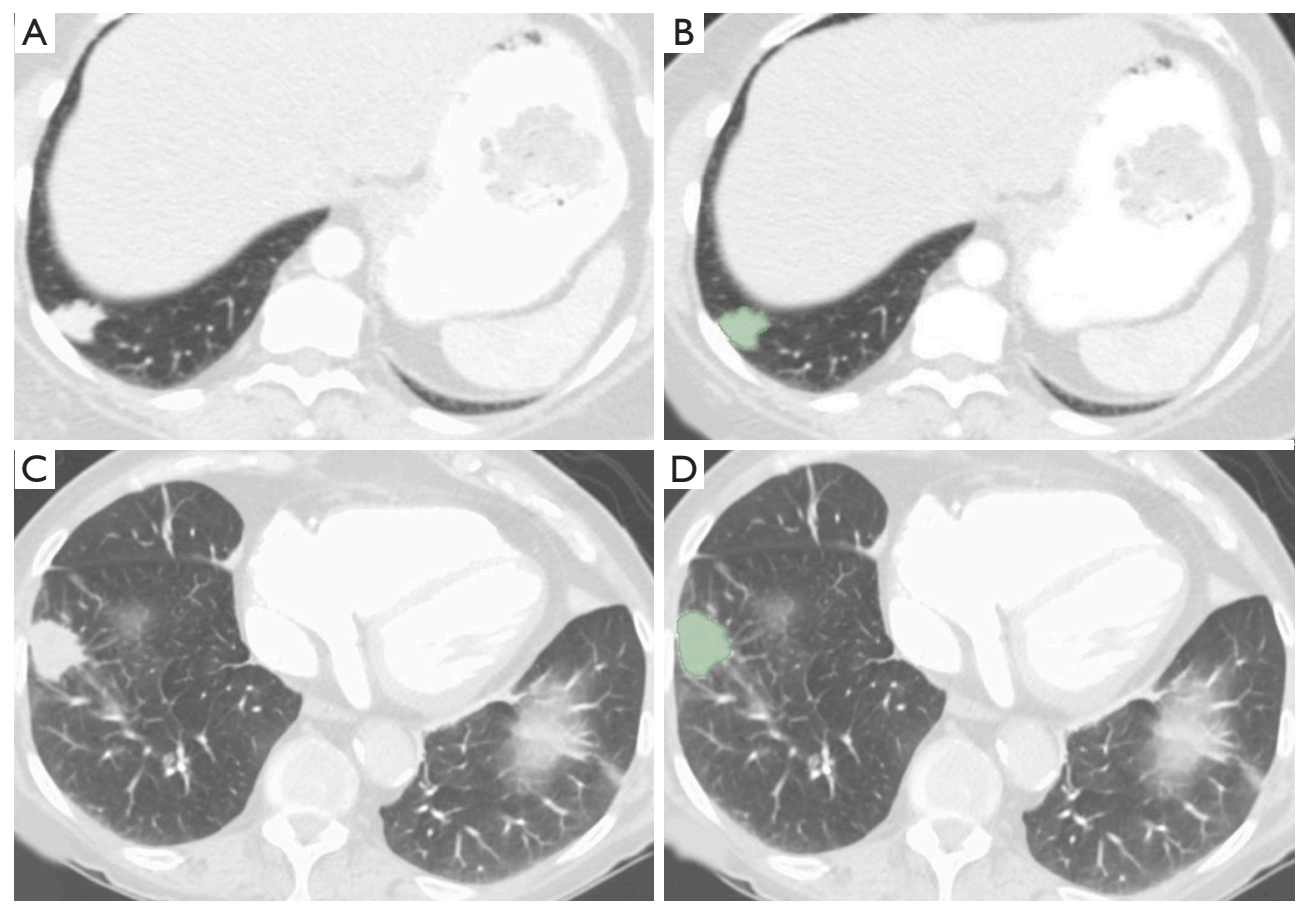

Figure 2 Transverse post-contrast CT image in lung window settings from a 67-year-old female shows irregular solid lesion in right lower lobe (stage 1 NSCLC) on unprocessed (A) and processed image (B). Another transverse CT image shows stage IV NSCLC from a 77-yearold female shows right lower lobe lesion on unprocessed (C) and processed (D) images.

operating characteristics (ROC) area under the curve was generated for clinical, imaging, and radiomic features. For comparing the radiomic features, two-tailed Student's $t$-test was used. A $\mathrm{P}$ value of less than 0.05 was considered statistically significant.

\section{Results}

The patient demographics are described in Table 1 .

\section{Comparison of stage IV vs. stages I-III combined (Table 2)}

Between stage IV and combined stages I-III, there was significant difference in forty (40/113) radiomic features by Pearson correlation $(\mathrm{P}=0.04-<0.0001)$ and in $26(26 / 113)$ radiomic features by $\mathrm{ROC}(\mathrm{P}=0.04-<0.009)$ [AUC 0.71 (95\% CI: 0.59-0.85)]. The common radiomic features that were different included, mean, variance, 10percentile, energy, run entropy, interquartile range, cluster prominence and tendency, complexity contrast, correlation, imc1/2, MCC, gray level variance, non-uniformity and small area emphasis. Complexity was the strongest predictor of differentiating stage IV versus other stages $(\mathrm{P}=0.009$,
Nagelkerke $\left.\mathrm{R}^{2}=0.18\right)$. None of the clinical (0/6) or imaging $(0 / 3)$ features were significantly different on Pearson correlation or ROC (AUC 0.54, P=0.3-0.9: AUC 0.61, $\mathrm{P}=0.1-0.9$ ).

Multivariate logistic regression analysis (Table 3) was performed by including selective radiomic features (including complexity, run entropy, variance, cluster tendency, grey level variance B, MCC, busyness). The backward conditional model based only on radiomic features correctly differentiated $73 \%$ of stage IV from other stages (sensitivity $88 \%$, specificity $54 \%$ ), slightly increasing the sensitivity compared to the univariate model. After adding clinical, imaging features to the radiomic features to multivariate logistic regression models there was differentiation $82 \%$ of stage IV from other stages (sensitivity $88 \%$, specificity $76 \%$ ). The logistic regression models were statistically significant (backward: conditional $\mathrm{P}=0.022$, Nagelkerke $\mathrm{R}^{2}=0.26$ ).

For probability variables (radiomics, clinical, imaging), ROC analysis showed higher AUC value for radiomic (AUC 0.67, $\mathrm{P}=0.026$ ) compared to clinical (AUC 0.57, $\mathrm{P}=0.3$ ) and standard imaging features (AUC 0.54, $\mathrm{P}=0.6$ ) in differentiating for stage IV from other stages. 
Table 1 Clinicopathologic and semantic imaging features of patients with BRAF-mutated NSCLC stratified by stage

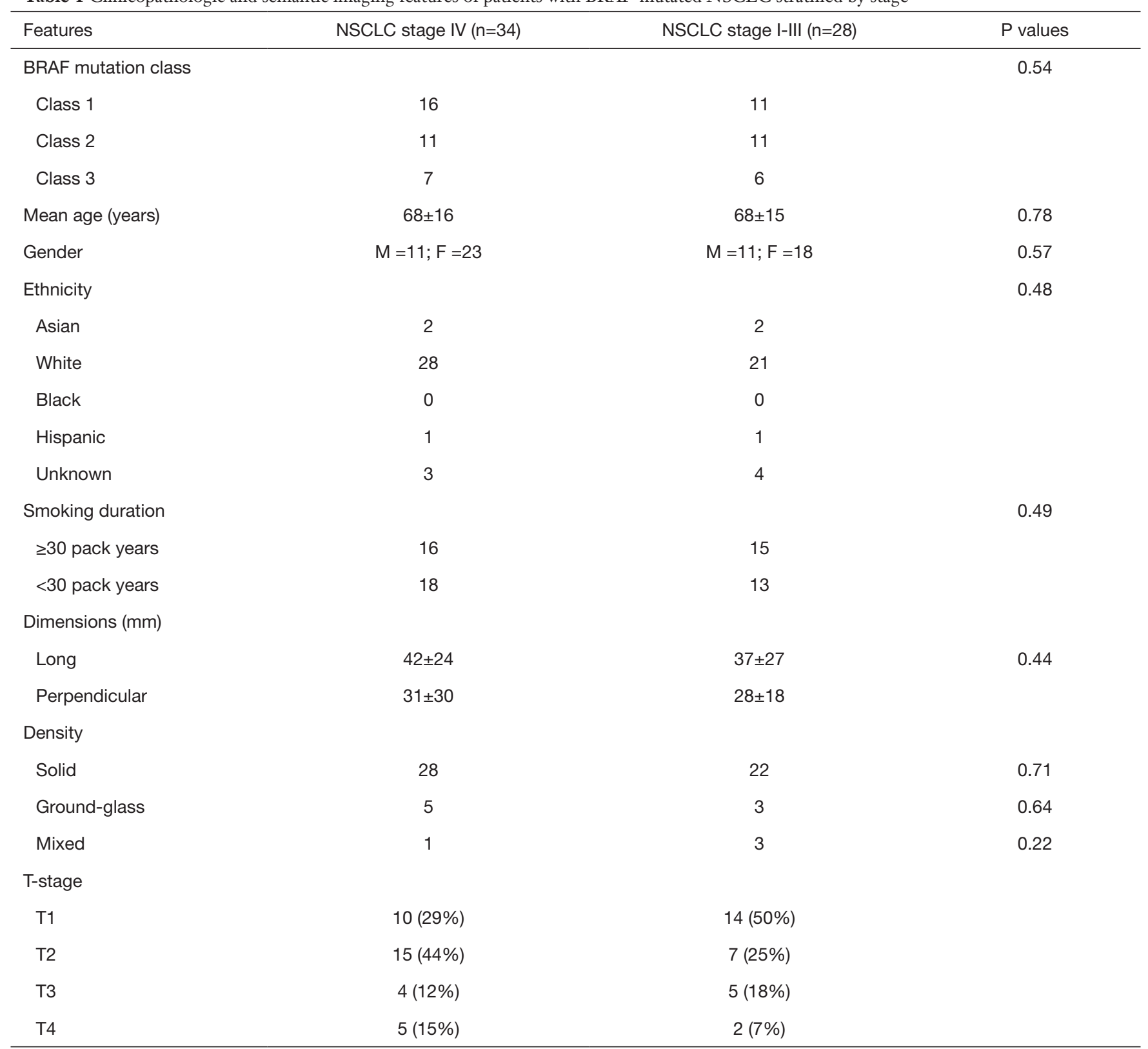

NSCLC, non-small cell lung cancer.

Comparison of stage IV vs. stage I-III separately (Table 4)

There was progressive increase in differences in radiomic features of the primary tumor with increase in stage of cancer. The highest difference was between stage IV and stage I and least between stage IV and stage III. Based on Pearson correlation, there were differences in $72 / 113$ radiomic features in primary tumor between stage $\mathrm{I}$ and IV, compared to $33 / 113$ between stage 2 and 4 and only in
1/113 between stage III and stage IV. Whereas based on ROC, there were differences in 84/113 of primary tumor radiomic features in stage I, 5/113 in stage II and 4/113 in stage III when compared to primary tumor in stage IV.

None of the clinical (best AUC 0.60; 0.62) or imaging (best AUC $0.60 ; 0.57$ ) features of primary tumor were significantly different for differentiating stage IV from stage II or III respectively $(\mathrm{P}=0.3-0.9)$. Whereas between stage 
Table 2 AUC values for radiomic features for stage IV vs. other stages

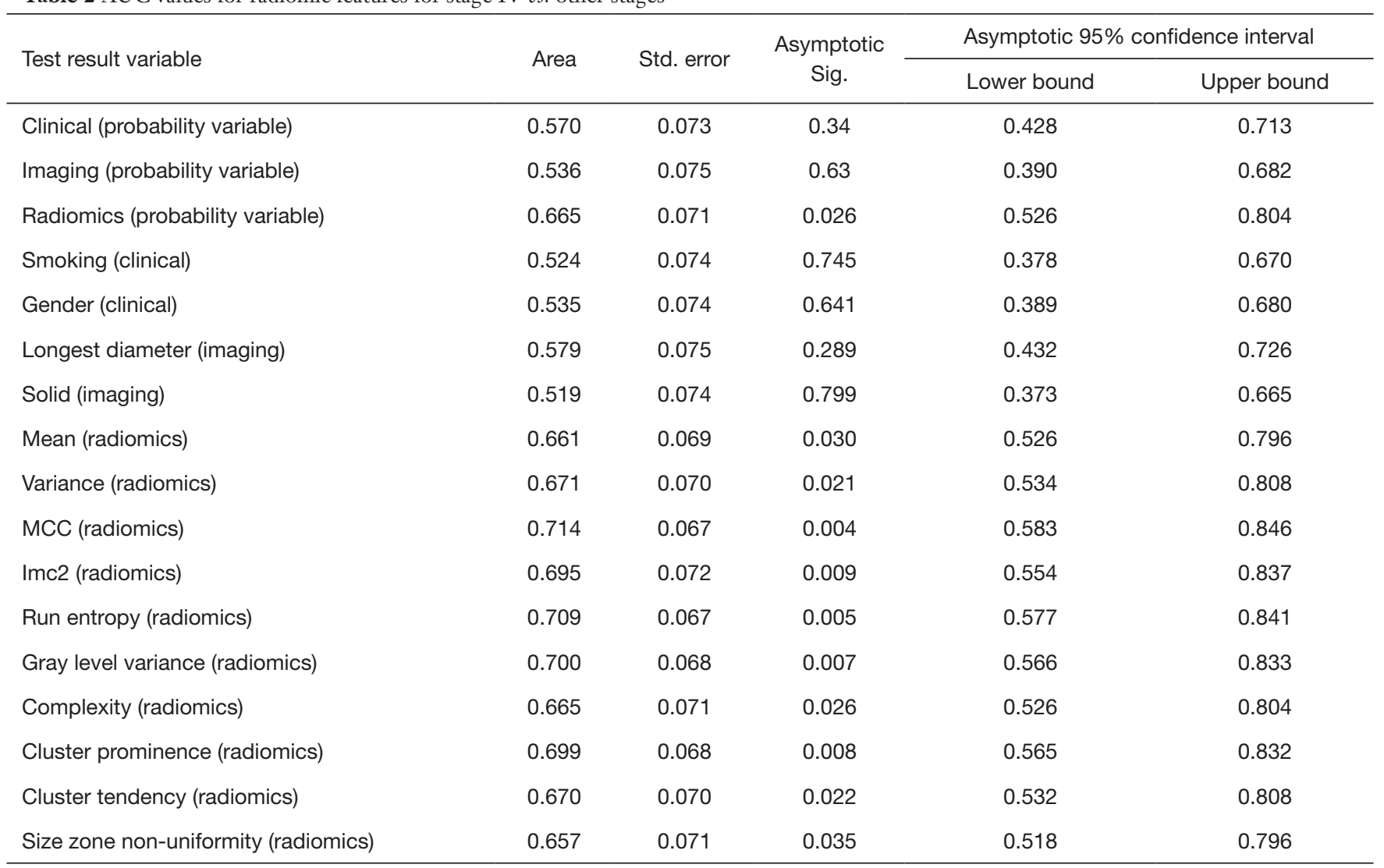

I and stage IV none of the clinical features (AUC 0.60, $\mathrm{P}=0.2-0.7$ ) were different, and $1 / 3$ imaging features (AUC $0.82, \mathrm{P}=0.006-0.9)$ was significantly different.

\section{Discussion}

Our study demonstrates that CT radiomic features of primary tumor in $B R A F$-mutated lung cancers are significantly different between TMN stages I, II, III, and IV. In comparison, commonly assessed tumor imaging features such as size, location, or density as well as common clinical characteristics related to age, sex, and smoking history could not differentiate between different stages of lung cancer. Similarly, we found that the highest accuracy in differentiation was between stage I and stage IV lung cancers (AUC 0.94 with 72 differentiating radiomic features), followed by between stage II and stage IV lung cancers (AUC 0.77 with 33 features), and finally between a stage III and stage IV lung cancer (AUC 0.71 with just 1 feature). The most important differentiating radiomic features included variance, energy, run entropy, interquartile range, cluster prominence and tendency, complexity contrast, correlation, gray level variance, non-uniformity and small area emphasis.

In our study, only $15 \%$ of stage IV $B R A F$-mutated lung cancers were $\mathrm{T} 4$ while most stage IV lung cancers (73\%) were T1 or T2. This apparent discordance may be explained by differences in underlying tumor biology, wherein clonal mutations within a tumor population increases the propensity for metastasis. A recent study on breast cancer metastasis have shown similar findings, with a non-linear relationship between cancer size and metastasis potential (22). The authors postulate a parallel model for explaining metastases whereby at the time of original tumor presentation, a parallel process is occurring in underlying cancer stem cell populations, which confers aggressivity in the cancer cells. Under this model, there is not necessarily a stepwise progression of metastatic disease from primary cancer, to local lymph nodes, and then to distant organs. On the contrary, the potential for metastasis is an intrinsic property of the tumor stem cell population, and clonal mutations conferring aggressivity may occur early in the 
Table 3 Logistic regression analysis with variable number of predictors for stage IV vs. combined stages I-III

\begin{tabular}{|c|c|c|c|c|c|c|c|c|c|}
\hline Step & Predictor & $\mathrm{B}$ & S.E. & Wald & df & Sig. & $\operatorname{Exp}(B)$ & \multicolumn{2}{|c|}{$95 \% \mathrm{Cl}$ for $\operatorname{EXP}(\mathrm{B})$} \\
\hline \multirow[t]{7}{*}{ Step 1} & ClusterTendency & -0.026 & 0.043 & 0.373 & 1 & 0.541 & 0.974 & 0.896 & 1.059 \\
\hline & MCC & -4.055 & 4.268 & 0.903 & 1 & 0.342 & 0.017 & 0.000 & 74.513 \\
\hline & GrayLevelVariance & -0.004 & 0.051 & 0.006 & 1 & 0.936 & 0.996 & 0.902 & 1.100 \\
\hline & Busyness & -0.468 & 0.552 & 0.720 & 1 & 0.396 & 0.626 & 0.212 & 1.846 \\
\hline & Complexity & -0.002 & 0.003 & 0.824 & 1 & 0.364 & 0.998 & 0.992 & 1.003 \\
\hline & Variance & 0.000 & 0.000 & 0.349 & 1 & 0.555 & 1.000 & 1.000 & 1.001 \\
\hline & Constant & 5.616 & 4.645 & 1.462 & 1 & 0.227 & 274.868 & & \\
\hline \multirow{4}{*}{ Step 2} & Busyness & -0.455 & 0.529 & 0.742 & 1 & 0.389 & 0.634 & 0.225 & 1.787 \\
\hline & Complexity & -0.003 & 0.002 & 1.372 & 1 & 0.241 & 0.997 & 0.993 & 1.002 \\
\hline & Variance & 0.000 & 0.000 & 0.363 & 1 & 0.547 & 1.000 & 1.000 & 1.001 \\
\hline & Constant & 5.770 & 4.234 & 1.857 & 1 & 0.173 & 320.506 & & \\
\hline \multirow[t]{3}{*}{ Step 3} & ClusterTendency & -0.031 & 0.039 & 0.640 & 1 & 0.424 & 0.970 & 0.899 & 1.046 \\
\hline & MCC & -4.748 & 3.676 & 1.668 & 1 & 0.197 & 0.009 & 0.000 & 11.674 \\
\hline & Busyness & -0.465 & 0.527 & 0.779 & 1 & 0.377 & 0.628 & 0.223 & 1.765 \\
\hline \multirow{3}{*}{ Step 4} & Busyness & -0.528 & 0.523 & 1.019 & 1 & 0.313 & 0.590 & 0.212 & 1.644 \\
\hline & Complexity & -0.002 & 0.002 & 1.087 & 1 & 0.297 & 0.998 & 0.995 & 1.002 \\
\hline & Constant & 5.013 & 2.904 & 2.979 & 1 & 0.084 & 150.283 & & \\
\hline \multirow[t]{4}{*}{ Step 5} & MCC & -5.659 & 3.486 & 2.636 & 1 & 0.104 & 0.003 & 0.000 & 3.231 \\
\hline & Busyness & -0.556 & 0.522 & 1.136 & 1 & 0.287 & 0.573 & 0.206 & 1.595 \\
\hline & Complexity & -0.002 & 0.001 & 3.735 & 1 & 0.053 & 0.998 & 0.995 & 1.000 \\
\hline & Constant & 5.546 & 2.753 & 4.057 & 1 & 0.044 & 256.140 & & \\
\hline \multirow[t]{3}{*}{ Step 6} & $\mathrm{MCC}$ & -4.612 & 3.303 & 1.949 & 1 & 0.163 & 0.010 & 0.000 & 6.441 \\
\hline & Complexity & -0.002 & 0.001 & 3.062 & 1 & 0.080 & 0.998 & 0.996 & 1.000 \\
\hline & Constant & 4.337 & 2.460 & 3.108 & 1 & 0.078 & 76.477 & & \\
\hline \multirow[t]{2}{*}{ Step 7} & Complexity & -0.003 & 0.001 & 6.852 & 1 & 0.009 & 0.997 & 0.995 & 0.999 \\
\hline & Constant & 0.998 & 0.393 & 6.455 & 1 & 0.011 & 2.712 & & \\
\hline
\end{tabular}

The logistic regression analysis with 1-7 radiomic features demonstrate no significant difference in overall sensitivity and specificity. Steps 1 to 7 analysis with decreasing number of radiomic features (step 1 with 7 and step 7 with only 1 feature). 
Table 4 AUC values for radiomic features for stage IV vs. stage I

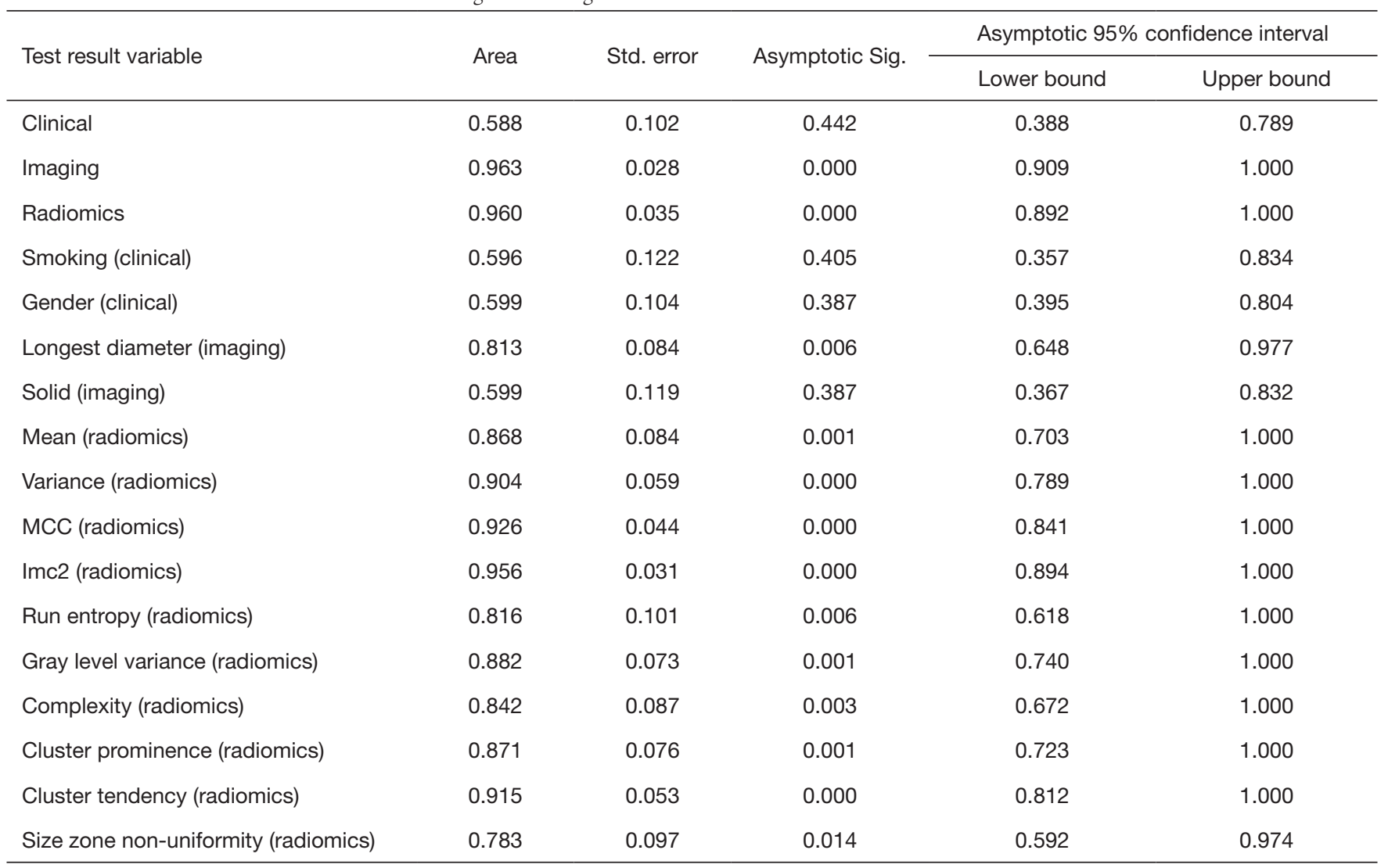

course of disease irrespective of local progression (6). Lending support to this model are several recent molecular studies in breast (23), pancreatic (24), melanoma (25), and colon cancer (26). Our results suggest that radiomic features of the primary lung tumors may helpful in capturing these biological differences, whether due to subtle differences in density or vascularity, which were previously beyond the scope of visual interpretation.

Advances in molecular research have established that tumors are more genetically and phenotypically unstable when compared to their normal counterparts $(26,27)$. Furthermore, it has been established that the majority of secondary or metastatic tumors are clonal in origin $(28,29)$. It is therefore perhaps unsurprising that we see more radiomic differences between early stage tumors and advanced stage tumors, providing further support to the notion of increased tumor heterogeneity as cancers progress along their life cycles. As tumor cells invade the stroma, multiple additional microscopic changes need to occur in the background, including angiogenesis and organization of vascular supply (30). Our results demonstrate that radiomic features can potentially tap into these features, which cannot be semantically captured during conventional image interpretation. Radiomic features can play an important role in noninvasively characterizing primary lung cancers and predict biologic aggressivity and metastatic potential.

Current cancer treatment relies on the resection of primary tumor and the prevention of metastasis, with much emphasis on distant metastasis given its direct association with mortality in patients (30). Current TNM and AJCC staging systems rely on cancer dimensions, local invasiveness, and the presence of distant metastases as an estimate of prognosis. However, since metastatic potential is likely an intrinsic characteristic of the tumor stem cells, there is a potential for radiomic features to be included in staging of tumors and prognosis.

There is a paucity of studies on the utility of radiomic features in differentiating or predicting the TMN stage of primary lung cancers. Radiomic survival analyses of NSCLC patients have previously demonstrated that entropy, skewness, mean density, roundness, and gray-level nonuniformity were most significant predictive features of 
progression-free survival $(31,32)$. Other studies have found that entropy, kurtosis, range, and skewness, were significant features that can differentiate or are associated with EGFR mutation status in primary lung adenocarcinoma $(15,30)$. More work is needed in this field to validate our findings and establish a standardized set of radiomic features to be assessed in the routine staging of lung cancers.

To our knowledge, this is the first systematic analysis of the utility of radiomic features in the assessment of different TMN stages in primary lung cancer. Only contrastenhanced CT exams were included to assess for differential enhancement in our texture analysis, especially given the importance of angiogenesis and vascular organization in the growth and invasion of lung cancer (30). Excluding any post-treatment lung cancer patients in this study also eliminated confounders related to treatment.

Our study has several limitations, including a relatively small sample size involving a relatively rare driver mutation, $B R A F$. There is a question of applicability of our results to other lung cancers with different driver mutations such as EGFR and ALK. Absence of a readily available cohort of patients with other mutations precluded us from performing a comparison. We also do not have data related to treatment response and long term outcomes. Further work in a multi-institutional study with other driver mutations and correlations with treatment response and outcomes will be needed next to assess the generalizability and robustness of our results. Another limitation of our study applies to the variable CT section thickness $(1-3 \mathrm{~mm})$, which could affect the reproducibility of radiomic features although the distribution of different section thicknesses was even across different cancer stages. Another potential limitation is use of scanners from different vendors, that can impact the reproducibility of radiomic features, particularly of higher statistical order (33). Given the time and effort involved in lesion identification, segmentation, radiomic features extraction and analyses, it is not clear how and if radiomic features can be applied in routine clinical practice.

In conclusion, radiomic features of primary tumor on contrast-enhanced CT scans are different between early and advanced tumor stages in $B R A F$-mutated lung cancer. There is increased heterogeneity in terms of texture features between early and advanced tumors, a finding that is in line with current molecular conception of parallel clonal expansion of tumor cells and intrinsic metastatic potential. Radiomic features can serve as a non-invasive biomarker in the initial staging and surveillance of lung cancer, although more work is needed to confirm the validity and generalizability of these findings.

\section{Acknowledgments}

Funding: This research did not receive any specific grant from funding agencies in the public, commercial, or notfor-profit sectors.

\section{Footnote}

Reporting Checklist: The authors have completed the MDAR and STROBE reporting Checklist. Available at http:// dx.doi.org/10.21037/tlcr-20-347

Data Sharing Statement: Available at http://dx.doi. org/10.21037/tlcr-20-347

Conflicts of Interest: All authors have completed the ICMJE uniform disclosure form (available at http://dx.doi. org/10.21037/tlcr-20-347). Dr. IDJ reports personal fees from Boehringer Ingelheim, and AstraZeneca, personal fees from Foundation Medicine, personal fees from Array and Pfizer, grants from Array, Genentech, Pfizer, and Guardant Health, outside the submitted work; Dr. MKK reports grants from Riverain technologies, grants from Siemens Healthineers, outside the submitted work. Dr. SRD reports Merck, Pfizer, Bristol Mayer Squibb, Novartis, Roche, Polaris, Cascadian, Abbvie, Gradalis, Clinical Bay, Zai laboratories, Siemens Medical Solutions, Lunit INC, outside the submitted work. The other authors have no conflicts of interest to declare.

Ethical Statement: The authors are accountable for all aspects of the work in ensuring that questions related to the accuracy or integrity of any part of the work are appropriately investigated and resolved. The study was conducted in accordance with the Declaration of Helsinki (as revised in 2013). This study was reviewed and approved by the Partners Human Research Committee (Institutional review board protocol numbers 2019P000198, 2016P000767). Informed consent was waived by the IRB due to retrospective nature of the study.

Open Access Statement: This is an Open Access article distributed in accordance with the Creative Commons Attribution-NonCommercial-NoDerivs 4.0 International License (CC BY-NC-ND 4.0), which permits the noncommercial replication and distribution of the article with 
the strict proviso that no changes or edits are made and the original work is properly cited (including links to both the formal publication through the relevant DOI and the license). See: https://creativecommons.org/licenses/by-nc-nd/4.0/.

\section{References}

1. Siegel RL, Miller KD, Jemal A. Cancer statistics, 2019. CA Cancer J Clin 2019;69:7-34.

2. Rami-Porta R, Bolejack V, Crowley J, et al. The IASLC Lung Cancer Staging Project: Proposals for the Revisions of the T Descriptors in the Forthcoming Eighth Edition of the TNM Classification for Lung Cancer. J Thorac Oncol 2015;10:990-1003.

3. Zhang J, Gold KA, Lin HY, et al. Relationship between tumor size and survival in non-small-cell lung cancer (NSCLC): an analysis of the surveillance, epidemiology, and end results (SEER) registry. J Thorac Oncol 2015;10:682-90.

4. Goldstraw P, Chansky K, Crowley J, et al. The IASLC Lung Cancer Staging Project: Proposals for Revision of the TNM Stage Groupings in the Forthcoming (Eighth) Edition of the TNM Classification for Lung Cancer. J Thorac Oncol 2016;11:39-51.

5. Rami-Porta R, Asamura $\mathrm{H}$, Travis $\mathrm{WD}$, et al. Lung cancer - major changes in the American Joint Committee on Cancer eighth edition cancer staging manual. CA Cancer J Clin 2017;67:138-55.

6. Friberg S, Nyström A. Cancer Metastases: Early Dissemination and Late Recurrences. Cancer Growth Metastasis 2015;8:43-9.

7. Almendro V, Marusyk A, Polyak K. Cellular heterogeneity and molecular evolution in cancer. Annu Rev Pathol 2013;8:277-302.

8. de Bruin EC, McGranahan N, Mitter R, et al. Spatial and temporal diversity in genomic instability processes defines lung cancer evolution. Science 2014;346:251-6.

9. Yokota J. Tumor progression and metastasis. Carcinogenesis 2000;21:497-503.

10. Silvestri GA, Gonzalez AV, Jantz MA, et al. Methods for staging non-small cell lung cancer: Diagnosis and management of lung cancer, 3rd ed: American College of Chest Physicians evidence-based clinical practice guidelines. Chest 2013;143:e211S-e250S.

11. Nishino M, Hatabu H, Johnson BE, et al. State of the art: Response assessment in lung cancer in the era of genomic medicine. Radiology 2014;271:6-27.

12. Thawani R, McLane M, Beig N, et al. Radiomics and radiogenomics in lung cancer: A review for the clinician. Lung Cancer 2018;115:34-41.

13. Digumarthy SR, Padole AM, Lo Gullo R, et al. CT texture analysis of histologically proven benign and malignant lung lesions. Medicine (Baltimore) 2018;97:e11172.

14. Digumarthy SR, Padole AM, Rastogi S, et al. Predicting malignant potential of subsolid nodules: can radiomics preempt longitudinal follow up CT? Cancer Imaging 2019;19:36.

15. Digumarthy SR, Padole AM, Gullo RL, et al. Can CT radiomic analysis in NSCLC predict histology and EGFR mutation status? Medicine (Baltimore) 2019;98:e13963.

16. Shi L, He Y, Yuan Z, et al. Radiomics for Response and Outcome Assessment for Non-Small Cell Lung Cancer. Technol Cancer Res Treat 2018;17:1533033818782788.

17. Avanzo M, Stancanello J, Pirrone G, et al. Radiomics and deep learning in lung cancer. Strahlenther Onkol 2020. doi: 10.1007/s00066-020-01625-9.

18. Planchard D, Smit EF, Groen HJM, et al. Dabrafenib plus trametinib in patients with previously untreated BRAFV600E-mutant metastatic non-small-cell lung cancer: an open-label, phase 2 trial. Lancet Oncol 2017;18:1307-16.

19. Paik PK, Arcila ME, Fara M, et al. Clinical characteristics of patients with lung adenocarcinomas harboring BRAF mutations. J Clin Oncol 2011;29:2046-51.

20. Mendoza DP, Dagogo-Jack I, Chen T, et al. Imaging characteristics of BRAF-mutant non-small cell lung cancer by functional class. Lung Cancer 2019;129:80-4.

21. Dagogo-Jack I, Martinez P, Yeap BY, et al. Impact of BRAF Mutation Class on Disease Characteristics and Clinical Outcomes in BRAF-Mutant Lung Cancer. Clin Cancer Res 2019;25:158-65.

22. Sopik V, Narod SA. The relationship between tumour size, nodal status and distant metastases: on the origins of breast cancer. Breast Cancer Res Treat 2018;170:647-56.

23. Hosseini H, Obradović MMS, Hoffmann M, et al. Early dissemination seeds metastasis in breast cancer. Nature 2016;540:552-8.

24. Rhim AD, Mirek ET, Aiello NM, et al. EMT and dissemination precede pancreatic tumor formation. Cell 2012;148:349-61.

25. Werner-Klein M, Scheitler S, Hoffmann M, et al. Genetic alterations driving metastatic colony formation are acquired outside of the primary tumour in melanoma. Nat Commun 2018;9:595.

26. Heppner GH, Miller FR. The cellular basis of tumor progression. Int Rev Cytol 1998;177:1-56. 
27. Strauss BS. Hypermutability and silent mutations in human carcinogenesis. Semin Cancer Biol 1998;8:431-8.

28. Popper HH. Progression and metastasis of lung cancer. Cancer Metastasis Rev 2016;35:75-91.

29. Yamamoto N, Yang M, Jiang P, et al. Determination of clonality of metastasis by cell-specific color-coded fluorescent-protein imaging. Cancer Res 2003;63:7785-90.

30. Ganeshan B, Miles KA, Young RCD, et al. In search of biologic correlates for liver texture on portal-phase CT. Acad Radiol 2007;14:1058-68.

31. Ganeshan B, Abaleke S, Young RCD, et al. Texture

Cite this article as: Padole A, Singh R, Zhang EW, Mendoza DP, Dagogo-Jack I, Kalra MK, Digumarthy SR. Radiomic features of primary tumor by lung cancer stage: analysis in $B R A F$ mutated non-small cell lung cancer. Transl Lung Cancer Res 2020;9(4):1441-1451. doi: 10.21037/tlcr-20-347 analysis of non-small cell lung cancer on unenhanced computed tomography: initial evidence for a relationship with tumour glucose metabolism and stage. Cancer Imaging 2010;10:137-43.

32. Shen TX, Liu L, Li WH, et al. CT imaging-based histogram features for prediction of EGFR mutation status of bone metastases in patients with primary lung adenocarcinoma. Cancer Imaging 2019;19:34.

33. Traverso A, Wee L, Dekker A, et al. Repeatability and reproducibility of radiomic features. Int J Radiat Oncol Biol Phys 2018;102:1143-58. 